tom of which is hard, can not furnish perfect protection to a coccyx one and a half inches long. The cut which we see in medical journals, showing the ischia resting on the saddle, while the tip of the coccyx is raised clear above the top of the pubic bones, is incorrect and misleading, for when the spine is erect, the tip of the coccyx comes nearly to a level with the pubic arch. The tuberosities of the ischia and the gluteal muscles should rest firmly on the saddle; but the soft parts in the pubic arch, the sacrosciatic ligaments, and the coccyx, must be free from pressure. The saddle should be too soft to bruise, and too firm to give. The best padding is wool felt. Hair is not good, because it soon becomes displaced, packs down and gets hard. The pneumatic pad is not as good as hair, because it is too hard when well inflated, and, when only partly filled, pressure at one point displaces all the air at that point, allowing the part making the most pressure to come in contact with the hard structure of the saddle. It is also liable to burst, or get punctured.

\section{THE FAUCIAL TONSIL.}

ITS SPHERE AS AN AGENT OF SYSTEMIC INFECTION.

Presented to the Section on Laryngology and Otology, at the Forty-ninth Annual Meeting of the American Medical Association, held at Denver, Colo., June 7-10, 1898.

BY GILLIFORD B. SWEENY, M.D. PITTSBLRG, PA.

The faucial tonsil must be regarded as occupying a very humble position in the human body, either from a histologic or a physiologic standpoint. In fact, the only function of any importance that can be attributed to it is that of leucocytogenesis. Of the entire glandular organism concerned in this work, the tonsil probably occupies the least important part. Its relation to phonation or sexual nutrition need be mentioned only to be dismissed, as every intelligent observer will testify that its absence in no way interferes with the former nor does its presence enhance the latter. $A_{8}$ an agent for the secretion of fluid intended to facili. tate agglutination, it is observed that it is no more active than the mucous membrane which surrounds it and replaces it when removed. The tonsil may be said to be most nearly normal when practically absent from the faucial cayity. It is only when it becomes the seat of morbid phenomena that it requires our serious attention.

It is not the purpose of this paper to dwell at length upon the individual diseases occurring in the tonsil, but rather to refer to them incidentally, especially when considering systemic manifestation having their origin in a morbid state of the tonsil and which in their ultimate results are far-reaching and often most disastrous. We submit for your consideration the following proposition: That the tonsil, while in a state of inflammation, is a frequent and common source of morbid processes which, ultimately, affect the entire human economy. Fortunately the scientific advances of the past few years yield considerable information which goes far toward aiding in establishing the truth of this proposition. We are no longer in doubt as to how the system is primarily attacked in diphtheria. Systemic infection, systemic symptoms must and do invariably follow local morbid processes, which often obtain their hold upon the body through the vulnerability of the organ first attacked. The same may be said of scarlatina, follicular tonsillitis, and, in our judgment, the same occurs in rheumatic fever, in many forms of tuberculosis, and in typhoid fever. That the faucial cavity is a most readily accessible point of attack in infectious diseases is easily understood when we consider the gross anatomic proportions of this region. From its position it may be compared to the smaller and constricted portion of a funnel situated at an abrupt angle. All the agents of respiration and deglutition must come in immediate contact with it in their passage back and forth. However mild the inflammatory process, whether acute or chronic, the tonsil becomes a hotbed for infection, and readily propagates and distributes the morbid products to adjacent and remote organs. Its anatomic construction so closely binds it to the vascular and lymphatic systems, that we are apt to overlook the local and initial manifestation and regard the secondary systematic invasion as the primary morbid process. The immediate result of repeated inflammation of the tonsil, terminating as it does in early life in hyperplasia, or in the mature subject, in true hypertrophy, is familiar to us all. Interfering with nasal respiration it produces the "mouth breather," with its train of deleterious results. Improperly filtered, moistened and warm air is hastily conveyed to the lungs, oxygenation is seriously interfered with, which promptly displays its ill effects in the dull, listless eye, impaired mentality, the pallid complexion, the contracted chest, and the anemic and often wasted muscular organism. Hypostatic congestion in the nasal tract is followed by interference with the normal functions of the organs of sight, smell and hearing, and encourages the hypertrophic process to extend indefinitely in all directions. None can deny the statement that the tonsil offers special facilities for the propagation of the diphtheritic bacillus; failing to admit this, how can we account for the fact that the vast majority of cases originate here? We can not generalize and say that it is because of the susceptibility of mucous tissues, for how really few cases originate in the nostril, the eye, or other equally accessible points.

I am fully convinced that not only is the tonsil a vulnerable point for diphtheria, but that when in a morbid state it becomes often a culture-medium for the propagation of germs which otherwise would perish before gaining access to the body of their host. The same is true to even a greater degree in those two other highly contagious and infectious diseasesscarlatina and follicular tonsillitis. While diphtheria may primarily attack some other organ than the tonsil. scarlatina certainly very seldom, and infectious follicular tonsillitis never, selects any other organ as a point of initial invasion. We are now able, I believe, to establish almost beyond doubt the infectious nature of rheumatism. One can not fail to observe the analogy in the systemic symptoms of follicular tonsillitis and acute rheumatism. While we have not yet been able to isolate a germ strictly characteristic of rheumatism, yet in this disease we find in the blood streptococci and staphylococci, which characterize follicular tonsillitis and are found in the tonsil when affected in that disease.

The metabolic theory as to the causation of rheu. matism is certainly barren of the essential facts necessary to establish even its plausibility. To assume that defective assimilation under widely varying circumstances may produce a specific chemic agent- 
lactic acid--and that this in its turn becomes the cause of rheumatism, is simply building a theory upon premises unsupported by facts or phenomena. The nervous theory, as to its origin, has been in a manner evolved from the former, and is equally devoid of underlying facts.

Medical science is not peculiar in that she recognizes no facts that are not supported by intelligible phenomena; and these phenomena going to prove the infectious nature of rheumatism are not isolated nor conflicting.

Since rheumatism has invaded the system through the blood, as shown in the case of a mother inferting the child in utero, since the systemic manifestations resemble those so often recognized in infectious diseases as in gonorrhea, septicemia and pyemia, it is certainly natural that we should search along similar lines for other sources of its development. Jaccoud, the eminent Frenchman, not only rezards the tonsil as being the usual channel by which rheumatism attacks the body but believes that under favorable systemic circumstances any abraded surface may prove an open gateway for infection. Clinical study of the features of acute rheumatism strongly suggests: defective elimination, chiefly renal and cutaneous, and a specific bacterium, which, having gained access to the body, is fostered and nourished by the products of retarded elimination while it wages war upon the normal leucocyte. This hypothesis will be found to be compatible with all the clinical phenomena of the disease and in conformity with other similar diseases whose etiology has been established.

Turning to the consideration of tuberculosis, while we find hardly a tissue of the body which enjoys immunity from its devastating ravages, there is certainly no class of tissue that suffers more often than the glandular, whether we consider that attenuated form of tuberculosis which displays itself in the strumous diathesis and which we find wandering about at pleasure, to the remote portions of the body, devastating all classes of tissue, from the hair follicle to the powerful bony framework of the body, or whether we turn to those more directly fatal forms of tuberculosis attacking the lungs and alimentary tract, we find that the glandular system plays an important part in admitting the tubercular bacillus into the body and in furnishing soil well adapted for its development and propagation. No observant physician can fail to observe the promptness with which the parotid, submaxillary and cervical glands become infected in all inflammatory conditions of the tonsil. It can not be again said that this same infectious process may easily and naturally extend to the bronchial and mesenteric glands and become the agent of infection of the adjacent organs. Different eminent observers have found that in a given number of tuberculous subjects but a small percentage of cases exhibited the tubercular bacillus in the tonsil, and therefore conclude that it is rarely the organ first attacked. We well know that tubercular infection takes place readily at any point where there is a solution of continuity. To conclude that the tonsil must be a very frequent agent to be an active one in the causation of tuberculosis is certainly not a fair deduction, especially in the absence of data showing just where and how infection did take place. Conclusive proof in that direction, we believe, must contemplate the furnishing of evidence tending to show that in persons affected with tonsillar disease but a small percentage developed tuberculosis. When such investigation is instituted it will be found that a very considerable percentage of cases of tuberculosis invade the system through the open gateway to the lymphatic system-the tonsil.

Lack of time prevents our going into elaborate detail in the consideration of the means of infection in typhoid and malarial fevers. That the one is due to a specific bacillus, while the other is characterized by hematozoa and plasmodia, whose exact natures have not yet been fully determined, we are aware. Moreover, we also know that of all the influences tending to spread both these diseases no other agent plays as important a part as drinking water. What has been already said regarding infection through the tonsil in other diseases may, with equally good reason, be said of both these fevers. We would not urge that every person having unhealthy tonsils must necessarily be infected in this manner because he has partaken of contaminated water. Intestinal infection can not be regarded as being very common, otherwise in a city with such water-supply as we have in Pittsburg - my native city - we would look for one universal and uninterrupted epidemic of typhoid fever. We would only claim that the tonsil, being an open gateway for reception of all kinds of disease germs, is as plausible a point for infection as Peyer's patches or Brunner's glands. While it is beyond the scope of this paper to consider the treatment of tonsillar affections, and while medicinal agents usually yield very satisfactory results, we can not refrain from criticising the disposition to indulge in the surgical coquetries in cases of hypertrophy of the tonsils by the use of injections, electrolysis or chemic caustics. Radical extirpation by means of the tonsillotome is here the method par excellence for disposing of the morbid condition with the least possible amount of pain and with every assurance of the mcst satisfactory results.

DISCUSSION.

Dr. Richards-Dr. Sweeney speaks of the desirability of removing the gland in order to prevent eystemic infection. I do not see how it is possible in most cases to remove all of the gland. If any crypts are left there is always the possibility of systemic infection again occurring. At the Philadelphia meeting last year the point was made that the adhesion to the anterior pillar should always be separated. Since then I have followed this method with considerable satisfaction. In spite of this I have been mortified that in a few cases there have been recurrent attacks of tonsillitis. So that now I am inclined to hedge when asked if patients will ever have an attack of tonsil. litis again. I always remove all the tonsil I can, but do not promise that when any crypts remain an attack of tonsillitis will never occur. It is not probable, but it may take place.

Dr. Coulter-My position in reference to the method of removal of the tonsil is quite well known. My paper, I apprehend, was the cause of the discussion which took place on this subject at the Philadelphia meeting. I do not believe the operation of tonsillectomy can be done by any possible method of operating other than that of a bloodless dissection by means of the cautery. You can by this method remove every follicle of the gland, and you can then promise the patient that there will not be a return of tonsillitis. Any operation which merely amputates the gland, leaves still more open this gateway to the system.

Dr. STucky questions advisability of attempting total extirpation of the tonsil. If one-half or two-thirds of the gland is removed, the remaining portion becomes so atrophied as to cause no future trouble.

Dr. BARNHILL, Indianapolis--I think in the operative treat. ment of tonsils that we should, as elsewhere, use our judgment and follow no one plan. In cases of children, the gland having no adbesions, projects sufficiently to be caught deeply with the McKenzie tonsillotome, and the desired result can thus be quickly and satisfactorily attained. But in many cases of diseased tonsil in the adult the tonsil is small, hard, adherent and does not project sufficiently to be caught deeply enough to cure 
the disease for which the operation is performed. And in such cases the tonsillotome is inefficient, and it seems to me that the operation suggested by Dr. Coulter would here be a good one. I have never believed it necessary to remove more of a tonsil than that which will include the bottoms of all the crypts. However, I do not believe the body will euffer any for complete removal, for the tonsil is a part of the general lymphatic system, and the several hundred other lymphatic glands of the body can readily carry on the work done by the tonsils. I think Dr. Coulter should carefully describe and widely distribute the method of his operation, for I am sure the profession does not understand its minutiæe.

\section{EPISTAXIS.}

A SIMPLE AND EFFICIENT MEANS FOR ITS CONTROL.

\section{BY BOYD CORNICK, M.D.}

$$
\text { KNICKERBOCKER, TEXAS. }
$$

Recurrent or prolonged nosebleeding occurs sufficiently often in general practice to occasionally prove a nightmare to those practitioners who must rely on the means of checking it which are recommended by most authorities.

Having witnessed, twenty-two years ago, the ineffectual efforts of a specialist to check the hemorrhage in a case of unilateral epistaxis, I naturally felt no little concern when, a few years later, I was called on to arrest a bleeding from the nose of some twenty hours' duration. The means finally resorted to (with success) was a strong astringent injection, by means of a postnasal syringe, the tip of which was inserted through the mouth into the upper pharynx, behind the velum palati, the fluid flooding the floor of both nasal passages, and flowing out of both nostrils anteriorly. But there is a better and simpler method than this, if I may judge from its prompt success in some half dozen severe cases encountered since then.

So late and so good an authority as Struempell's "Text-book of Medicine" (revised edition) recommends plugging the nares with absorbent cotton. In all the cases in which $I$ have known this measure to be adopted the result was a failure. Perhaps it was not done secundem artem. And perhaps, with so unsatisfactory material, this method requires more skill than most practitioners-and some specialistspossess.

My treatises on general surgery, on the other hand, lay no little stress on internal remedies, and fall back on the plugging of the posterior nares with Bellocq's canula as a last resort. Meantime, if they refer to an attempt to plug the anterior nares, it is with the recommendation that a quill, or a section of a gum catheter, be enclosed in the plug, and that a solution of perchlorid of iron be used to first saturate the plug. Of these recommendations the first is needless bother, and the second produces needless irritation.

There may be constitutional conditions predisposing to nosebleed, which call for internal medication. Plethora or cachexia, coexisting with epistaxis, calls each for its own appropriate treatment. Nor is it my purpose to deal in this paper with those cases of intermittent nosebleed, occurring in adolescence, without debilitating the patient, nor with that class of patients in later life where, because of contracted kidney and arterial sclerosis, an occasional profuse epistaxis acts, as a safety-valve in the steam engine, by preventing a degree of intra-arterial pressure which threatens apoplexy.

The class of cases which I have encountered, though generally presenting evidence of some constitutional disturbance of the normal condition of the blood, or of the normal blood-pressure, has likewise given evidence of the existence of a localized solution of continuity in the nasal mucous membrane itself. In all such cases, were the lesion situated externa!ly, so as to be easily visible and readily gotten at, what authority would lay stress on internal medication to constringe the arterioles, as an aid to checking the hemorrhage? What surgeon would propose astringent or styptic applications to the bleeding site?

It is because the site of the lesion, in most cases of even persistent epistaxis, while not easily visible, is nevertheless as easily subject to compression as though on the external surface of the body that, in all the cases I have encountered within the last fifteen years or more, compression of the bleeding site has been as readily accomplished, and has been as promptly successful, as though it had been located on the external surface.

All that I have found necessary has been to fashion with a pair of seissors a dry plug of prepared sponge, in size and length comparable with the little finger of a 12-year-old-boy. This should be carefully soaked in boiled water, to free it of grit, squeezed dry to free it of unnecessary fluid, and inserted its full length, gently, along the floor of the bleeding nostril. No styptic is necessary; it would be needlessly irritant. The expansive pressure of the soft sponge against the bleeding site, increased by the coagulation of a few drops of blood in its interstices, will check the bleeding at once. Remove it in twelve hours. Under no circumstance let it remain longer than twenty-four. Melted vaselin containing .5 per cent. of carbolic acid applied with a medicine-dropper in liberal quantities is the only local treatment called for afterward.

\section{PELVIC MASSAGE.}

\section{BY LLEWELLYN P. BARBOUR, M.D.} BOLLDER, COLO.

The recent papers in the Journal of the AmerICan Medical asfociation discussing massage have been of much interest and of much worth to the general practitioner. It is to be regretted that this valuable remedial measure is not more widely appreciated by all who deal with pathologic conditions of the female pelvis. There is nothing in its technic that may not be done by the general practitioner with a good knowledge of the anatomy and physiology of the pelvis, and with ordinary dexterity. My attention was forcibly called to the subject six years ago by Dr. Dudley, of the New York Post-Graduate School, and the results I have since obtained have been a source of much satisfaction to me. I will report two recent cases, not because they surpass the success of the previous ones, but because they are recent, and because they serve as an example of what can be accomplished.

Case 1.-Mrs. Y. This patient first applied to me for treatment in July, 1897. Three years previously she had miscarried at three months. I could not learn the cause of the abortion. She was quite sick afterward, evidently from septic infection. She has suffered from menstrual pains since-not before-and somewhat from "bearing-down" sensations. She is troubled with constipation; has not been pregnant since the abortion, but is anxious to become so.

An examination revealed a retroverted, prolapsed and subinvoluted uterus, glued firmly in its position by inflammatory adhesions. Befors coming to me this woman had consulted two physicians-one an eminent gynecologist-and both ha 